\title{
Revealing the structure of the lensed quasar Q $0957+561$
}

\section{Accretion disk size}

\author{
C. Fian ${ }^{1}$, E. Mediavilla ${ }^{2,3}$, J. Jiménez-Vicente ${ }^{4,5}$, V. Motta ${ }^{6}$, J. A. Muñoz ${ }^{7,8}$, D. Chelouche ${ }^{9}$, P. Goméz-Alvarez ${ }^{10}$, \\ K. Rojas ${ }^{11}$, and A. Hanslmeier ${ }^{12}$
}

${ }^{1}$ School of Physics and Astronomy and Wise Observatory, Raymond and Beverly Sackler Faculty of Exact Sciences, Tel-Aviv University, Tel-Aviv, Israel e-mail: carinafian@mail.tau.ac.il

2 Instituto de Astrofísica de Canarias, Vía Láctea s/n, La Laguna 38200, Tenerife, Spain

3 Departamento de Astrofísica, Universidad de la Laguna, La Laguna 38200, Tenerife, Spain

4 Departamento de Física Teórica y del Cosmos, Universidad de Granada, Campus de Fuentenueva, 18071 Granada, Spain

5 Instituto Carlos I de Física Teórica y Computacional, Universidad de Granada, 18071 Granada, Spain

${ }^{6}$ Instituto de Física y Astronomía, Universidad de Valparaíso, Avda. Gran Bretaña 1111, Playa Ancha, Valparaíso 2360102, Chile

7 Departamento de Astronomía y Astrofísica, Universidad de Valencia, 46100 Burjassot, Valencia, Spain

8 Observatorio Astronómico, Universidad de Valencia, 46980 Paterna, Valencia, Spain

9 Haifa Research Center for Theoretical Physics and Astrophysics, University of Haifa, Haifa, Israel

10 FRACTAL S.L.N.E., Calle Tulipán 2, Portal 13, 1A, 28231 Las Rozas de Madrid, Spain

11 Institute of Physics, Laboratoire d'Astrophysique, Ecole Polytechnique Fédérale de Lausanne (EPFL), Observatoire de Sauverny, 1290 Versoix, Switzerland

12 Institute of Physics (IGAM), University of Graz, Universitätsplatz 5, 8010 Graz, Austria

Received 5 November 2020 / Accepted 6 July 2021

\begin{abstract}
Aims. We aim to use signatures of microlensing induced by stars in the foreground lens galaxy to infer the size of the accretion disk in the gravitationally lensed quasar Q $0957+561$. The long-term photometric monitoring of this system (which so far has provided the longest available light curves of a gravitational lens system) permits us to evaluate the impact of uncertainties on our recently developed method (controlled by the distance between the modeled and the experimental magnitude difference histograms between two lensed images), and thus to test the robustness of microlensing-based disk-size estimates.

Methods. We analyzed the well-sampled 21-year GLENDAMA optical light curves of the double-lensed quasar and studied the intrinsic and extrinsic continuum variations. Using accurate measurements for the time delay between the images A and B, we modeled and removed the intrinsic quasar variability, and from the statistics of microlensing magnifications we used a Bayesian method to derive the size of the region emitting the continuum at $\lambda_{\text {rest }}=2558 \AA$.

Results. Analysis of the Q $0957+561 R$-band light curves show a slow but systematic increase in the brightness of the B relative to the A component during the past ten years. The relatively low strength of the magnitude differences between the images indicates that the quasar has an unusually big optical accretion disk of half-light radius: $R_{1 / 2}=17.6 \pm 6.1 \sqrt{M / 0.3 M_{\odot}}$ lt-days.
\end{abstract}

Key words. gravitational lensing: micro - quasars: individual: Q 0957+561 - accretion, accretion disks

\section{Introduction}

The temporal changes in brightness of the images of a gravitationally lensed quasar can be described as a combination of time-delay-correlated (intrinsic variability of the source) and uncorrelated (gravitational microlensing) variations, and their analysis has important applications in cosmology such as the determination of time delays to infer the Hubble constant (Rusu et al. 2020; Wong et al. 2020; Birrer et al. 2020), the estimate of peculiar velocities (Mediavilla et al. 2016), and in the study of quasar structure (Chang \& Refsdal 1979, 1984; see also Kochanek 2004 and Wambsganss 2006). Up to now it has been impossible to spatially resolve the emitting regions of quasars, even with the largest available optical telescopes. The random distribution of compact objects such as stars in the foreground lens galaxy induce uncorrelated flux anomalies in the multiple quasar images, which can help us overcome these difficulties and thus can be used to extract information about both the source and the lens itself. These (de)magnifications produced by microlenses depend strongly on the angular size of the source, with smaller emission regions showing bigger changes in the brightness, while larger sources result in smoother light curves (Mosquera \& Kochanek 2011; Blackburne et al. 2011, 2014). Over the past years, quasar microlensing has therefore become a powerful tool to study the continuum emission regions of quasars by measuring and modeling the time-variable flux ratios between lensed images (e.g., Motta et al. 2012; Blackburne et al. 2014, 2015; Jiménez-Vicente et al. 2012, 2014; Mosquera et al. 2009, 2013; Mediavilla et al. 2015; Muñoz et al. 2016; Fian et al. 2016, 2018; Morgan et al. 2018).

Q $0957+561$ was discovered by Dennis Walsh in 1979 . At a redshift of $z_{s}=1.41$, the quasar is lensed into two bright point sources. The separation between images is $\sim 6^{\prime \prime}$, and 
Table 1. Time delay measurements from the literature.

\begin{tabular}{lc}
\hline \hline$\Delta t$ (days) & Reference \\
\hline $423 \pm 6$ & Pelt et al. (1996) \\
$404 \pm 26$ & Schild \& Thomson (1997) \\
$417 \pm 3$ & Kundić et al. (1997) \\
$422.6 \pm 0.6$ & Oscoz et al. (2001) \\
$429.9 \pm 1.2$ & Ovaldsen et al. (2003) \\
$417.09 \pm 0.07$ & Colley et al. (2003) \\
$417 \pm 2$ & Shalyapin et al. (2008) \\
\hline
\end{tabular}

several studies (Pelt et al. 1996; Oscoz et al. 1996, 1997, 2001; Schild \& Thomson 1997; Kundić et al. 1997; Ovaldsen et al. 2003; Colley et al. 2003; Shalyapin et al. 2008) reported a time delay of $\sim 14$ months (see Table 1) between the two images (with A being the leading component). The low microlensing variability and the lack of microlensing events in the early light curves of the first known gravitational lens Q 0957+561 prevented determinations of the quasar's accretion disk size (Schmidt \& Wambsganss 1998; Wambsganss et al. 2000). After combining new optical monitoring data with previously published data, Hainline et al. (2012) reported a new microlensing event and thus demonstrated the return of long-timescale, uncorrelated variability in the light curves of Q $0957+561$. In total, they used $\sim 15$ years of photometric monitoring (although with rather large gaps) to constrain the size of the optical accretion disk. In this work, we conducted a statistical analysis of an extended data set of 21 years of photometric monitoring, which currently features the longest available light curves of a gravitationally lensed quasar. Our aim is to use this large amount of monitoring data to study the existence of possible microlensing events and to place improved constraints on the size of the continuum-emitting region (hereafter referred to as accretion disk, although the contribution of additional continuum components may be non-negligible; Chelouche et al. 2019). We followed the single-epoch method combined with the flux ratios of a large enough source in the quasar in order to be insensitive to microlensing and to establish the baseline for no microlensing magnification (see, e.g., Metcalf \& Zhao 2002; Mediavilla et al. 2009). The broad emission lines' cores (arising from the narrowline region), the mid-IR (emitted by the dusty torus), and the radio emitting regions (radio jet and lobes) of quasars should all be large enough to average out the effects of microlensing and allow the determination of the "intrinsic" flux ratios between images (Kochanek 2004). Flux ratios detected at radio wavelengths are considered as the most robust, because the sources are believed to be much larger than the Einstein radius $\eta_{0}$. However, radio flux ratios are measurable only for a small subsample of lensed quasars bright enough at radio wavelengths (about one out of five known systems, see Sluse et al. 2013), and other proxies of the unbiased intrinsic flux ratios have to be found. Furthermore, Guerras et al. (2013) also suggested that the cores of the broad emission lines are not subject to a large microlensing variation and can be used as a baseline for no microlensing.

In the present work, we extended the single-epoch method to more than 1000 epochs in the available light curves, thereby increasing the statistical significance. We used the optical R-band light curves obtained from the GLENDAMA project (Gil-Merino et al. 2018) to infer microlensing flux variability and radio data from the literature to estimate the baseline for no microlensing variability. After correcting for the relatively long time delay and the mean magnitude difference between the images, we find clear indications of slow microlensing variability in the residuals of the light curves over the past ten years. Following the methods described in Fian et al. (2016, 2018), we compared the histogram of microlensing magnifications obtained from the observations (corresponding to the monitoring time interval) with the simulated predictions of microlensing variability for sources of different sizes. This comparison allowed us to evaluate the likelihood of the different values adopted for the size. In the present study, apart from using the so far longest available light curves, we used a more rigorous method to estimate the accretion disk size, and, in addition, we evaluated the effect of different kinds of uncertainties on the size of the accretion disk.

The paper is organized as follows. In Sect. 2, we present the full combined light curves of images A and B of Q $0957+561$. We outline our modeling of the intrinsic variability and examine the flux ratios between the images in Sect. 3. Section 4 is devoted to the Bayesian source size estimation based on the statistics of microlensing magnifications. In Sect. 5, we present the results and discuss the impact of uncertainties on the size estimates. Finally, we give a brief summary in Sect. 6.

\section{Data}

The fluxes of the two images of Q $0957+561$ were monitored from 1996 February until 2016 May in the optical $R$-band (at $\lambda_{\text {rest }}=2558 \AA$ ) as a part of the Gravitational LENses and DArk MAtter (GLENDAMA) project (see Gil-Merino et al. 2018). They used the observations made at the $0.8 \mathrm{~m}$ telescope of the Instituto de Astrofísica de Canarias' (IAC) Teide Observatory (Oscoz et al. 1996, 1997, 2001, 2002; Serra-Ricart et al. 1999) during the first observing period (1996-2005). They later monitored the double quasar with the $2 \mathrm{~m}$ Liverpool Telescope (LT) at the Roque de los Muchachos Observatory from 2005 to 2016 (Gil-Merino et al. 2018). The data set consists of 1067 epochs of observation (i.e., 1067 nights), and the average sampling rate is once every seven days. In Table 2, we list the object's characteristics. In Fig. 1, an image of Q $0957+561$ is shown and, in Fig. 2 the 21-yr light curves of the images A and B are presented.

\section{Intrinsic variability and microlensing}

Quasars are time variable, and since the images of multiple lensed quasars arrive with relative delays ranging from hours to several years because of the different paths taken by their light, variability of the source can mimic flux ratio anomalies. However, studies of optical continuum variability in gravitationally lensed quasars have the advantage that one is usually able to disentangle intrinsic from extrinsic variability (e.g., Oscoz et al. 1996, 1997; Kundić et al. 1997; Paraficz et al. 2006; Goicoechea et al. 2008; Shalyapin et al. 2008). To analyze the light curves of Q $0957+561$ for the presence of extrinsic variations (i.e., microlensing), we first have to model and remove this variability that is intrinsic to the quasar itself. To accomplish that, we used the most recent time delay estimates (Shalyapin et al. 2008) and shifted the light curve of image B by -417 days. Thereafter, we corrected for the magnitude difference between the images using radio data from the literature, assuming that these data represent the true magnification ratios of the images in the absence of microlensing. The radio-emitting regions of quasars should provide a realistic baseline for no microlensing, as they are supposed to arise 
Table 2. Q 0957+561 characteristics.

\begin{tabular}{lcccccc}
\hline \hline $\begin{array}{l}\text { RA }\left({ }^{\prime \prime}\right) \\
(1)\end{array}$ & $\begin{array}{c}\text { Dec }\left({ }^{\prime \prime}\right) \\
(2)\end{array}$ & $\begin{array}{c}z_{s} \\
(3)\end{array}$ & $\begin{array}{c}z_{l} \\
(4)\end{array}$ & $\begin{array}{c}N \\
(5)\end{array}$ & $\begin{array}{c}\delta\left({ }^{\prime \prime}\right) \\
(6)\end{array}$ & $\begin{array}{c}r \text {-SDSS band } \\
(7)\end{array}$ \\
\hline $0 / 1.229$ & $0 /-6.048$ & 1.413 & 0.3562 & 2 & 6.26 & $17.5 / 16.9$ \\
\hline
\end{tabular}

Notes. Columns (1) and (2): Relative coordinates (right ascension and declination) of images A and B from the CASTLES Survey (https: //lweb.cfa.harvard.edu/castles/). Columns (3)-(4): Redshift of the quasar and the lens galaxy from the Gravitationally Lensed Quasar Database (https://research. ast . cam. ac . uk/lensedquasars/). Columns (5)-(6): Number of images and image separation from the CASTLES Survey. Column (7): $r$-SDSS band magnitudes of image A and image B (see Popović et al. 2021).

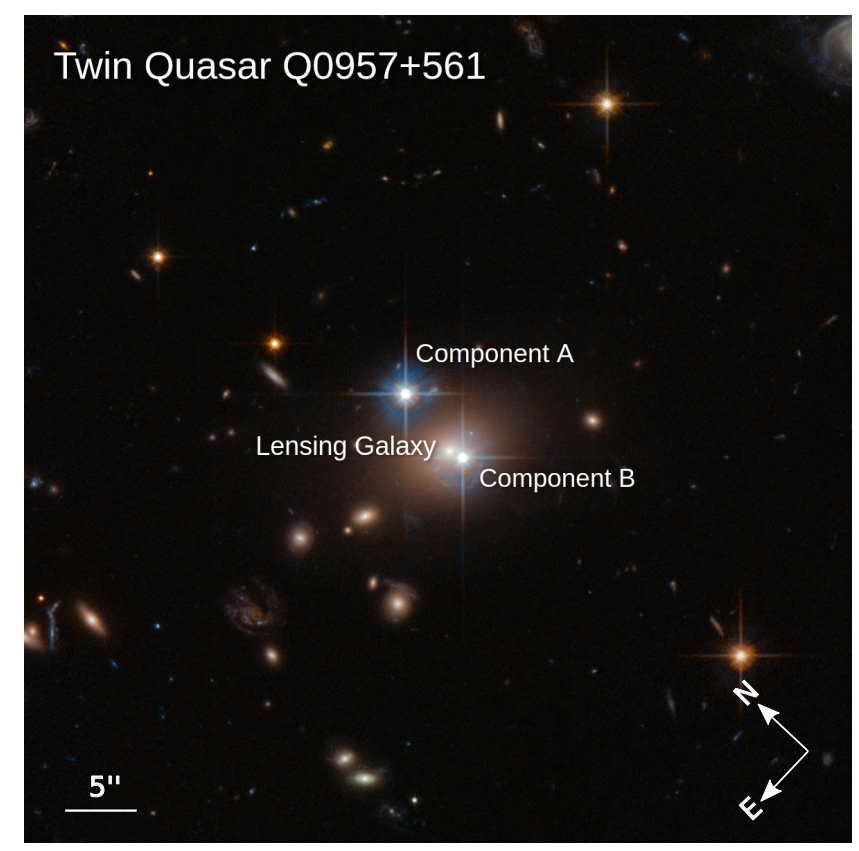

Fig. 1. Hubble image (https://esahubble.org/images/ potw1403a/) of the gravitationally lensed quasar Q $0957+561$ in the constellation Ursa Major. The two components are separated by $\sim 6^{\prime \prime}$, with image B being located close $\left(\sim 1^{\prime \prime}\right)$ to the lensing galaxy $\mathrm{G} 1$, which is a giant elliptical lying within a cluster of galaxies that also contributes to the lensing. The field of view $(\mathrm{FoV})$ is $\sim 1$ square arcmin.

from a large enough region to be insensitive to microlensing (see, e.g., Metcalf \& Zhao 2002; Mediavilla et al. 2009). We assume that the flux variations in image A are mainly intrinsic as light from this component passes far from the lens galaxy. Early studies (see, e.g., Schild \& Smith 1991) already attributed any differences in brightness between the A and B image to microlensing of the $\mathrm{B}$ component as the surface mass density of the lens galaxy is much lower at the position of component $\mathrm{A}$. Less than $0.05 \%$ (see Jiménez-Vicente et al. 2015a) of the mass is expected to be in compact objects at this distance from the lens galaxy, coupled with an Einstein crossing time of $\sim 12.4$ years (see Mosquera et al. 2011), which implies unlikely microlensing events for this quasar component. Light from the B component, however, passes through the lens galaxy (image B appears about $1^{\prime \prime}$ away from the center of G1; see Fig. 1), and the probability of microlensing by the densely packed stars in the lens galaxy is relatively high (Young 1981; Schild et al. 1990; Schild \& Smith 1991). As image A is expected to be unaffected by microlensing, it gives us an accurate history of the quasar's intrinsic brightness fluctuations. We obtain a source variability of $\sim 0.4 \mathrm{mag}$ over the total duration of photometric monitoring. In addition, we averaged the individual measurements into one-year bins and examined the mean and maximum changes in brightness during this time (see Fig. 3). We obtain a mean variation of $\sim 0.1$ mag year $^{-1}$ and a maximum variation of $\sim 0.2$ mag year $^{-1}$, respectively.

In this work, we studied two different cases: in the first case we performed a linear interpolation of image A's light curve to generate a set of photometric measurements at the same epochs of observations as those of the shifted light curve of image B. In the second case, we estimated the amplitude of the intrinsic variability by performing a single spline fitting to the A light curve (the image less prone to microlensing). Finally, we can subtract from the shifted light curve of image B the interpolated light curve/spline fitting of image A, creating a difference light curve (residuals) in which only the uncorrelated variability remains.

In Fig. 4, we present the time-delay shifted light curves of Q $0957+561$ together with the residuals calculated from $\Delta m_{B}=$ $m_{B}-m_{A(\text { fit })}-(m B-m A)_{\text {radio. }}$. From the upper panel in Fig. 4, we can see that during the first ten years (1996-2006) the light curves overlap well; then, a brightening of image B relative to image A is visible, which can be directly related to microlensing caused by stars in the lensing galaxy. Despite the similarity between the A and B light curves in this decade, some contribution of weak microlensing variability cannot be completely discarded. However, this is irrelevant because in our treatment we also consider the contributions of image A to microlensing in the simulated difference light curves. In the lower panel of Fig. 4, we can also clearly see the well-known quasi-constancy of the residuals of the B image between 1996 and 2006. In more recent years, higher variability took place (Hainline et al. 2012; Shalyapin et al. 2012; Gil-Merino et al. 2018) and the new results support the claim of Hainline et al. (2012) that a microlensing event occurred during these years. The duration of this microlensing event (starting in 2009 and lasting at least until 2015) is still unclear and further future monitoring to map the full extent of the event will be needed. During this sixyear range, the magnitude difference between the light curves increased from $\sim 0.1 \mathrm{mag}-0.2 \mathrm{mag}$. The low microlensing variability together with the long timescale are consistent with the relatively large optical quasar size of $\sim 12$ lt-days reported in Hainline et al. (2012).

\section{Bayesian source size estimation}

We use a quantitative Bayesian method together with our determinations of the microlensing magnification amplitude to estimate the accretion disk size in the Q $0957+561$ lensed quasar. The basic idea is to compare the histogram of microlensing magnification obtained from the observations (corresponding to the monitoring time interval) with the simulated predictions of microlensing variability for sources of different size (see Fian et al. 2016, 2018). 


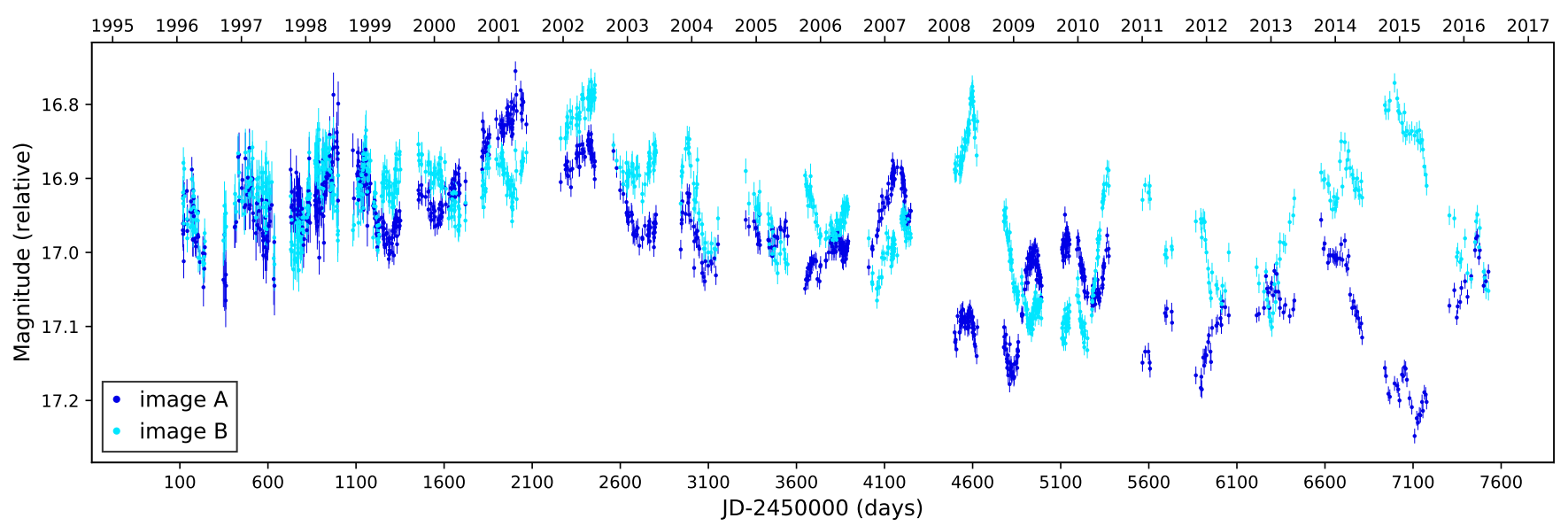

Fig. 2. Light curves of the lensed images A and B of the quasar Q $0957+561$ from 1996 February to 2016 May as obtained by the GLENDAMA project (see Gil-Merino et al. 2018). Horizontal axes show the Julian (bottom) and Gregorian (upper) dates.

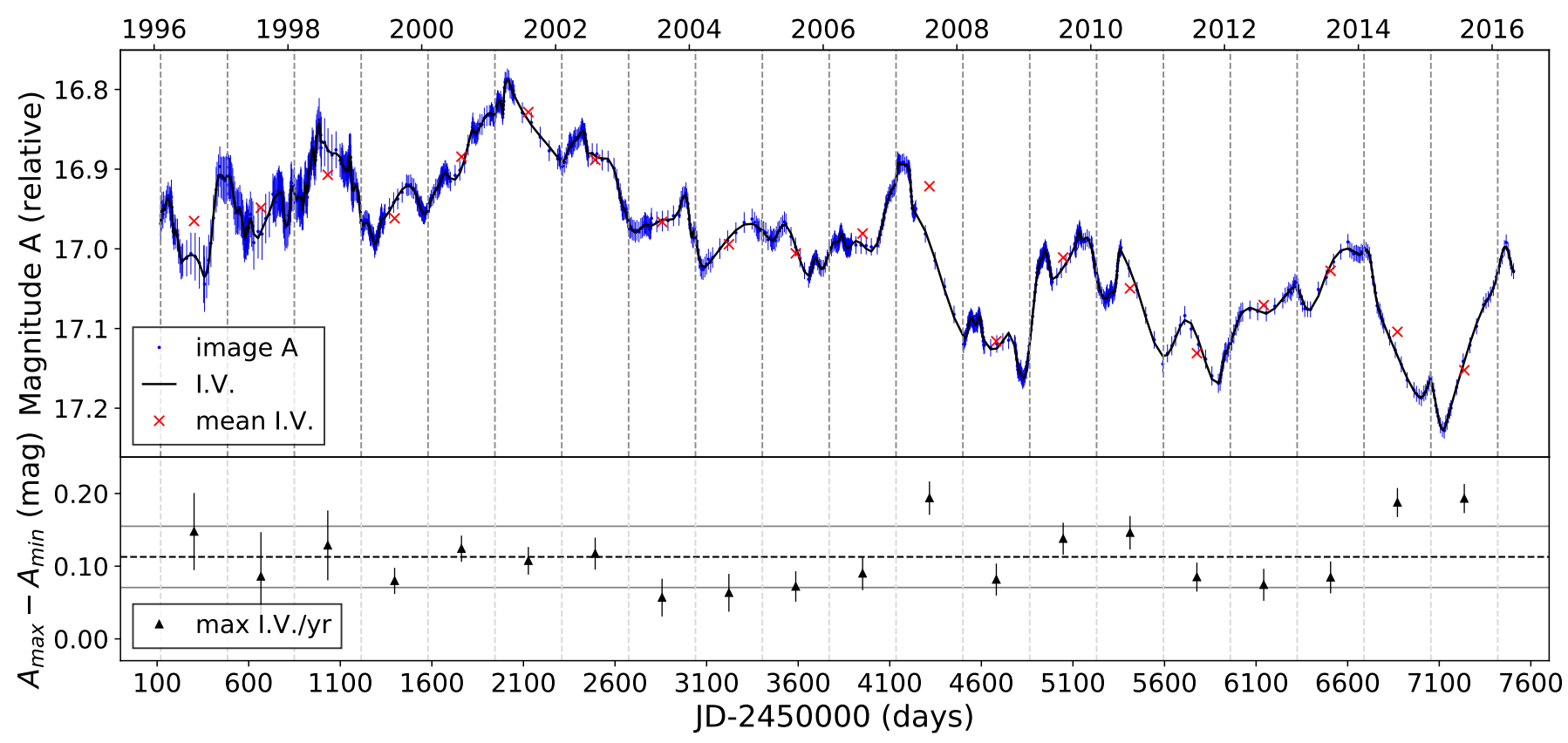

Fig. 3. Top: intrinsic variability (spline fitting to the A light curve) plus mean variability per year (red crosses). Bottom: maximum magnitude changes of the intrinsic variability per year.

\subsection{Simulated microlensing histograms}

To simulate the microlensing of extended sources, we use microlensing magnification maps created (for each quasar image) with the inverse polygon mapping method described in Mediavilla et al. (2006, 2011). Such maps show the microlensing magnification at a given source position and are determined by the local convergence, $\kappa$, and the local shear, $\gamma$, which can be obtained by fitting a singular isothermal sphere with an external shear $\left(\mathrm{SIS}+\gamma_{\mathrm{e}}\right)$ to the coordinates of the images. The values of $\kappa$ and $\gamma$ for images A and B (taken from Mediavilla et al. 2009) are listed in Table 3. A magnitude difference of $m_{B-A}=$ $-0.30 \mathrm{mag}$ was used for the macromodel, inferred from the average line-flux ratios of $\mathrm{Mg}$ II, C III], C IV, $\mathrm{N} \mathrm{V}$, and Ly $\alpha$ (taken from Goicoechea et al. 2005), consistent with the more recent estimate of the magnitude difference in $\mathrm{Mg}$ II reported by Motta et al. (2012). We used a surface mass density in stars of $\alpha=10 \%$ (Mediavilla et al. 2009) and generated $2000 \times 2000$ pixel $^{2}$ magnification maps with a resolution of 0.2 lt-days per pixel (much smaller than the size of the optical accretion disk of the quasar), spanning $17.4 \times 17.4$ Einstein radii $^{2}$. The value of the Einstein radius for this system is $3.25 \times 10^{16} \sqrt{M / 0.3 M_{\odot}} \mathrm{cm}=12.55 \sqrt{M / 0.3 M_{\odot}}$ lt-days at the source plane (Mosquera \& Kochanek 2011). We randomly distribute stars of a mass of $M=0.3 M_{\odot}$ across the microlensing patterns to create a microlens convergence of $10 \%$. The ratio of the magnification in a pixel to the average magnification of the map gives the microlensing magnification at the pixel and histograms of normalized to the mean maps deliver the relative frequency of microlensing magnification amplitude of a pixel-size source.

To model the structure of the unresolved quasar source, we considered a circular Gaussian intensity profile of size $r_{s}$, $I(R) \propto \exp \left(-R^{2} / 2 r_{s}^{2}\right)$. It is generally accepted that the specific shape of the source's emission profile is not important for microlensing flux variability studies, since the results are mainly controlled by the half-light radius rather than by the detailed source profile (Mortonson et al. 2005). The characteristic 


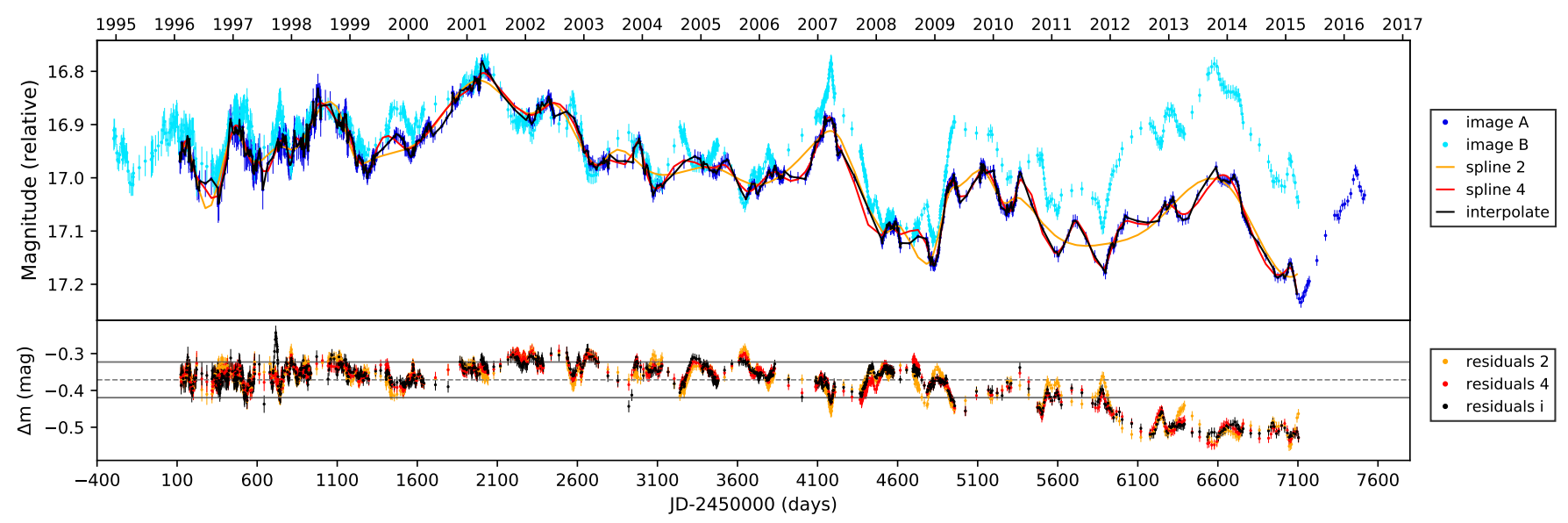

Fig. 4. Top: image A and B light curves of Q $0957+561$ in their overlapping region after shifting by the time delay. The linear interpolation of image A's light curve is shown in black, and two different models for the intrinsic variability of the quasar (splines with different knot steps fit to light curve A) are shown in red and in orange. To avoid confusion, we only display two out of four spline fittings. Bottom: differential microlensing variability of the light curve B compared to the linear interpolation (black)/spline fits (red and orange) to light curve A. The dashed horizontal line shows the mean value of the residuals. We note that the residuals have been corrected for the magnitude difference between the images using radio data.

Table 3. Lens model parameters.

\begin{tabular}{lcc}
\hline \hline Image & $\kappa$ & $\gamma$ \\
\hline A & 0.20 & 0.15 \\
B & 1.03 & 0.91 \\
\hline
\end{tabular}

size $r_{s}$ is related to the half-light radius, that is, the radius at which half of the light at a given wavelength is emitted, by $R_{1 / 2}=1.18 r_{s}$. As lengths are measured in Einstein radii, which scale as $R_{\mathrm{E}} \propto \sqrt{M}$, all calculated sizes can be rescaled accordingly for a different mean stellar mass. Finally, we convolve the magnification maps with Gaussians of 21 different sizes over a linear grid that spans from $r_{s}=0.5$ to 40.5 lt-days and after convolution, we normalize each map by its mean value. The histograms of the normalized map represent the histograms of the expected microlensing variability. Thus, we obtain 21 different microlensing histograms corresponding to different source sizes. The movement of a extended source across the magnification map (appearing as a network of high-magnification caustics separated by regions of lower magnifications) is equivalent to a point source moving across a map that has been smoothed by convolution with the intensity profile of the source. Large values or $r_{s}$ smear out the network of microlensing magnification caustics and reduce their dynamic range, thereby causing the histograms to become narrower. Finally, convolving the histograms of $\mathrm{B}$ with the histogram of $\mathrm{A}$, we build the microlensing difference histograms B-A for different values of $r_{s}$ to be compared with the observational histograms obtained from the light curves (see Sect. 4.2). We adopt a bin size of $0.05 \mathrm{mag}$ for the modeled and experimental microlensing histograms.

\subsection{Observed microlensing histograms}

The effect of a finite source size is that it smooths out the flux variations in the light curves of lensed quasars caused by stars in the galaxy. From the residual light curves that represent the differential (with respect to A, the image less prone to microlensing) microlensing of the $\mathrm{B}$ image, we obtained the microlensing variability histogram; this refers to the frequencies at which each microlensing amplitude appears in the microlensing variability light curves. In Fig. 5, we compare the B-A modeled magnification histograms corresponding to convolutions with sources of different sizes (dashed lines) with the experimental microlensing histograms.

\subsection{Method}

To study the likelihood of different values adopted for the size, we compare the microlensing histograms inferred from the model (corresponding to the convolutions with different source sizes) with the histogram of the data using the following statistic:

$P_{X}\left(r_{s}\right)=\sum_{i=1}^{N_{\text {bin }}} h_{X-B}^{i} \hat{h}_{X-B}^{i}\left(r_{s}\right)$,

where $h_{X-B}^{i}$ and $\hat{h}_{X-B}^{i}\left(r_{s}\right)$ are the observed and modeled histograms, and $N_{\text {bin }}$ is the number of bins. This histogram product is based on the distance between histograms (related to the Pearson correlation coefficient) and is a natural extension of the single-epoch method.

\section{Accretion disk size and impact of uncertainties}

To check the robustness of our microlensing based method used to estimate the size of the quasar's accretion disk, we study the impact of different uncertainties and sources of systematic errors.

\subsection{Uncertainties in fitting the intrinsic variability}

To study the importance of accurately fitting the intrinsic variability, we studied five different cases. In the first case, we performed a linear interpolation of image A's light curve and subtracted it from the shifted B light curve (see Fig. 4). Given the available data with high signal-to-noise ratio $(\mathrm{S} / \mathrm{N})$, we are able to produce a reasonable set of residuals (see lower panel in Fig. 4). In the other four cases, the idea was to fit one single spline representing the intrinsic variation of the quasar. For each 


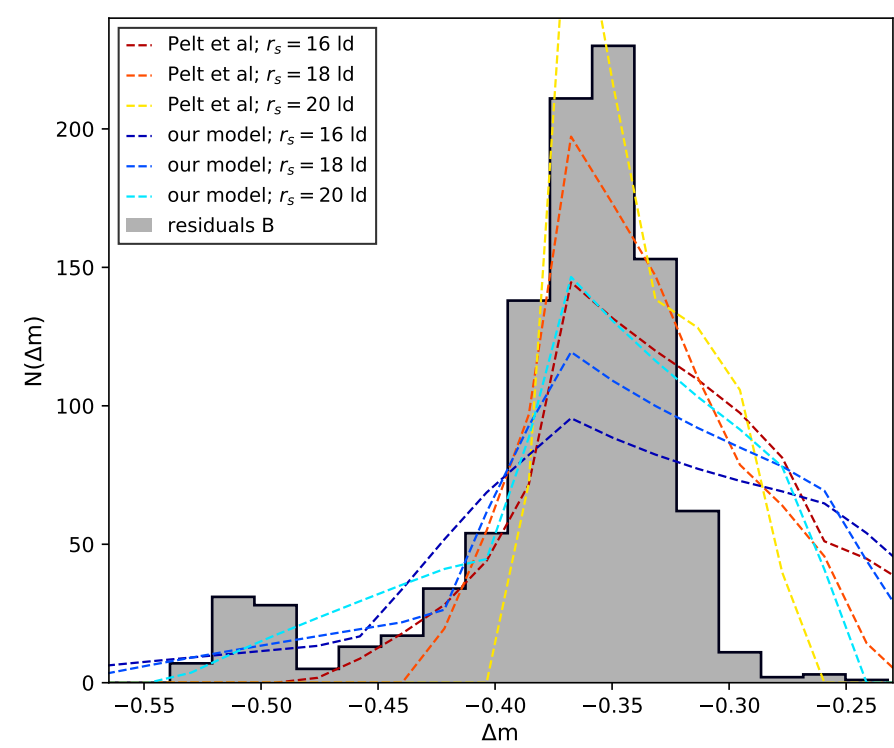

Fig. 5. Microlensing frequency distributions obtained from the observations, i.e, the difference light curve presented in the lower panel of Fig. 4 (gray histogram), and the simulated magnification maps (segmented lines). The various segmented lines correspond to convolutions of the simulated magnification maps with sources of different sizes (in blue shades for our macrolens model and in red shades for the model of Pelt et al. 1998).

Table 4. Accretion disk size using different models for the quasar's intrinsic variability.

\begin{tabular}{lcc}
\hline \hline I.V. model & $r_{s}$ (lt-days) & $R_{1 / 2}$ (lt-days) \\
\hline Interpolation & $14.9_{-10.4}^{+7.6}$ & $17.6_{-12.3}^{+9.0}$ \\
Spline 1 & $14.8_{-10.3}^{+7.7}$ & $17.5_{-12.2}^{+9.1}$ \\
Spline 2 & $14.8_{-10.3}^{+7.7}$ & $17.5_{-12.2}^{+9.1}$ \\
Spline 3 & $14.9_{-10.4}^{+7.6}$ & $17.6_{-12.3}^{+9.0}$ \\
Spline 4 & $14.9_{-10.4}^{+7.6}$ & $17.6_{-12.3}^{+9.0}$ \\
\hline
\end{tabular}

spline, we used a different smoothness (or flexibility), meaning we changed the initial spacings (in days) of the knots and repeated the same procedure as in the first case. From Fig. 4, we see that although the splines with the lowest knot steps (e.g., spline 1 and spline 2) cannot adequately capture the underlying structure of the data and do not fit fast changes in magnitude well, the resulting residuals look almost the same. Hence, neither underfitting nor overfitting produce significant changes in the residuals for light curves with moderate SNR. Producing histograms of the different sets of residuals and comparing them with the simulated histograms for different sizes of $r_{s}$, we obtain very similar results in all five cases (see Table 4).

\subsection{Uncertainties in the time delay}

Intrinsic variations in brightness records of gravitationally lensed quasars can be used to estimate global time delays between components (Refsdal 1964), and after several years of analyzing different sets of data from various telescopes, the scientific community appears to be converging on a time-delay value near 400 days (see Table 1). A serious problem for the time-delay estimation from the optical monitoring data had been
Table 5. Radio and BEL measurements from the literature.

\begin{tabular}{lcc}
\hline \hline $\mathrm{B} / \mathrm{A}$ & $\lambda$ & Reference \\
\hline $0.82 \pm 0.02$ & $13 \mathrm{~cm}$ & Gorenstein et al. (1988) \\
$0.79 \pm 0.05$ & $18 \mathrm{~cm}$ & Garrett (1990) \\
$0.752 \pm 0.028$ & $6 \mathrm{~cm}$ & Conner et al. (1992) \\
$0.723 \pm 0.044$ & $6 \mathrm{~cm}$ & Conner et al. (1992) \\
$0.76 \pm 0.03$ & $13 \mathrm{~cm}$ & Chartas et al. (1995) \\
$0.75 \pm 0.02$ & $18 \mathrm{~cm}$ & Garrett et al. (1994) \\
$0.74 \pm 0.02$ & 4 and 6 cm & Haarsma et al. (1999) \\
$0.759 \pm 0.007$ & Mg II (MMT) & Motta et al. (2012) \\
$0.787 \pm 0.022$ & Mg II (HST) & Motta et al. (2012) \\
$\sim 0.69$ & Several BELs & Motta et al. (2012) \\
\hline
\end{tabular}

Table 6. Accretion disk size using different radio and BEL ratios.

\begin{tabular}{ccc}
\hline \hline$B / A$ & $r_{s}$ (lt-days) & $R_{1 / 2}$ (lt-days) \\
\hline 0.69 & $13.1_{-8.6}^{+7.4}$ & $15.5_{-10.7}^{+8.7}$ \\
0.72 & $14.1_{-9.6}^{+8.4}$ & $16.6_{-10.3}^{+10.1}$ \\
0.73 & $14.5_{-10.0}^{+8.0}$ & $17.1_{-11.8}^{+9.4}$ \\
0.74 & $14.8_{-10.3}^{+7.7}$ & $17.5_{-12.2}^{+9.1}$ \\
0.75 & $15.4_{-10.9}^{+9.1}$ & $18.2_{-12.9}^{+10.9}$ \\
0.76 & $15.8_{-9.3}^{+8.7}$ & $18.6_{-11.0}^{+10.3}$ \\
0.77 & $16.4_{-11.9}^{+10.1}$ & $19.4_{-14.0}^{+11.9}$ \\
0.78 & $16.8_{-12.3}^{+9.7}$ & $19.8_{-11.4}^{+11.4}$ \\
0.79 & $17.1_{-12.6}^{+11.4}$ & $20.2_{-14.9}^{+13.5}$ \\
0.80 & $17.9_{-13.4}^{+10.6}$ & $21.1_{-15.8}^{+12.5}$ \\
0.81 & $18.3_{-11.2}^{+12.2}$ & $21.6_{-14.4}^{+14.9}$ \\
0.82 & $18.8_{-12.3}^{+11.7}$ & $22.2_{-14.5}^{+13.8}$ \\
\hline
\end{tabular}

the imperfect sampling, since brightness changes on time scales of days and weeks have been observed (Schild \& Thomson 1997). We check the influence of uncertainties in the time delay on the size estimates by adopting a value of $\Delta t=417$ days (taken from Shalyapin et al. 2008) and additionally shifting the light curves by $\pm 1 \sigma$ and $\pm 2 \sigma$, respectively. The relatively small uncertainties in the time delay (of the order of a few days) do not induce significant changes in the disk size measurement.

\subsection{Uncertainties in the baseline for no microlensing}

We studied the change of the accretion disk size when using different radio and emission line flux ratio measurements from the literature (see Table 5). In a spectroscopic analysis, Motta et al. (2012) used broad emission lines (BELs) to estimate the base for no microlensing, finding that the $\mathrm{B}-\mathrm{A}$ magnitude differences follow a decreasing trend toward the blue compatible with extinction. Toward the red, the flux ratios are fully consistent with the $\mathrm{B}-\mathrm{A}$ radio measurements (which are uncontaminated by the lens galaxy continuum and free from dust extinction). We found that the size measurements are sensitive to the baseline for no microlensing, in the sense that a flux ratio change of 0.1 results in a difference of $\sim 5$ lt-days (see Table 6 ). However, the uncertainties in the latest radio measurements are relatively small $(\sim 0.02$; see Haarsma et al. 1999), leading to a difference of only one lt-day. 
Table 7. Lens model parameters from Pelt et al. (1998).

\begin{tabular}{lcccc}
\hline \hline Image & $\kappa$ & $\gamma$ & $\Delta \kappa^{(*)}$ & $\Delta \gamma^{(*)}$ \\
\hline A & 0.22 & 0.17 & 0.02 & 0.02 \\
B & 1.24 & 0.90 & 0.21 & 0.01 \\
\hline
\end{tabular}

Notes. ${ }^{(*)}$ Difference compared to our model.

Table 8. Impact of uncertainties on the size estimates.

\begin{tabular}{lccc}
\hline \hline Source & $r_{s}$ (lt-days) & $R_{1 / 2}$ (lt-days) & $\Delta R_{1 / 2}{ }^{(a)}$ (lt-days) \\
\hline I.V. ${ }^{(b)}$ & $14.8_{-10.3}^{+7.7}$ & $17.5_{-12.2}^{+9.1}$ & -0.1 \\
$\Delta t$ & $14.8_{-10.3}^{+7.7}$ & $17.5_{-12.2}^{+9.1}$ & -0.1 \\
Baseline & $15.3_{-6.8}^{+5.1}$ & $18.1_{-8.0}^{+6.0}$ & +0.4 \\
Model & $10.6_{-6.1}^{+7.9}$ & $12.5_{-7.3}^{+9.3}$ & -5.1 \\
$\alpha^{(c)}$ & $18.3_{-11.8}^{+12.2}$ & $21.0_{-13.9}^{+14.4}$ & +3.4 \\
\hline
\end{tabular}

Notes. ${ }^{(a)}$ Deviation from the inferred disk size of $R_{1 / 2}=17.6$ lt-days (see dotted black line in Fig. 6). ${ }^{(b)}$ Intrinsic variability. ${ }^{(c)}$ Mass in stars.

\subsection{Uncertainties in the lens model}

The values of the convergence, $\kappa$, and shear, $\gamma$, at the location of each image are needed to compute the magnification maps from which the simulated microlensing histograms are obtained. These parameters are inferred from the macrolens model and may be affected by uncertainties. We checked for the robustness of our results with respect to the macromodel by comparing it with the lens parameters from Pelt et al. (1998) (see Table 7). After computing the magnifications maps for images A and $\mathrm{B}$, we repeat all of the calculations, obtaining a slightly smaller value for the half-light radius of the accretion disk $\left(R_{1 / 2}=12.5_{-7.2}^{+9.3}\right.$ lt-days $)$. The relationship of the errors in the macromodel with the uncertainty in the disk size is not simple and probably depends on the change in the magnification of the source.

\subsection{Uncertainties in fraction of mass in stars}

The amplitude of microlensing is sensitive to the local stellarsurface mass-density fraction (as compared to that of the dark matter at the image location see, e.g., Schechter \& Wambsganss 2002). Hence, the source size is sensitive to the stellar mass fraction $\alpha$. In Q $0957+561$, the two lensed images are located at very different radii from the center of the lens, resulting in different fractions of convergence in the form of stars. Jiménez-Vicente et al. (2015a) measured the stellar mass fraction at two different radii from a sample of 18 different lens system with available $R_{\mathrm{E}} / R_{\mathrm{eff}}$, where $R_{\mathrm{E}}$ is the Einstein radius and $R_{\text {eff }}$ is the effective radius of the lens galaxy. They found that the stellar-surface mass density is $\alpha \sim 0.3$ at a radius of $(1.3 \pm 0.3) R_{\text {eff }}$. We recomputed the magnification maps using a value $\alpha=0.3$ for image $\mathrm{B}\left(R_{\mathrm{E}} / R_{\mathrm{eff}}=1.29\right.$ for Q $0957-561$; see Fadely et al. 2010), and adopting a value of $\alpha=0$ for image A, which is equivalent to not using any magnification map for this image (due to the contribution of the cluster gravitational potential, this image is located far from the lens galaxy). We repeated all the calculations and obtained a slightly bigger value for the half-light radius of the accretion disk $\left(R_{1 / 2}=21_{-13.9}^{+14.4}\right.$ lt-days $)$. When $\alpha=0$ for image A, the B-A microlensing magnifica-

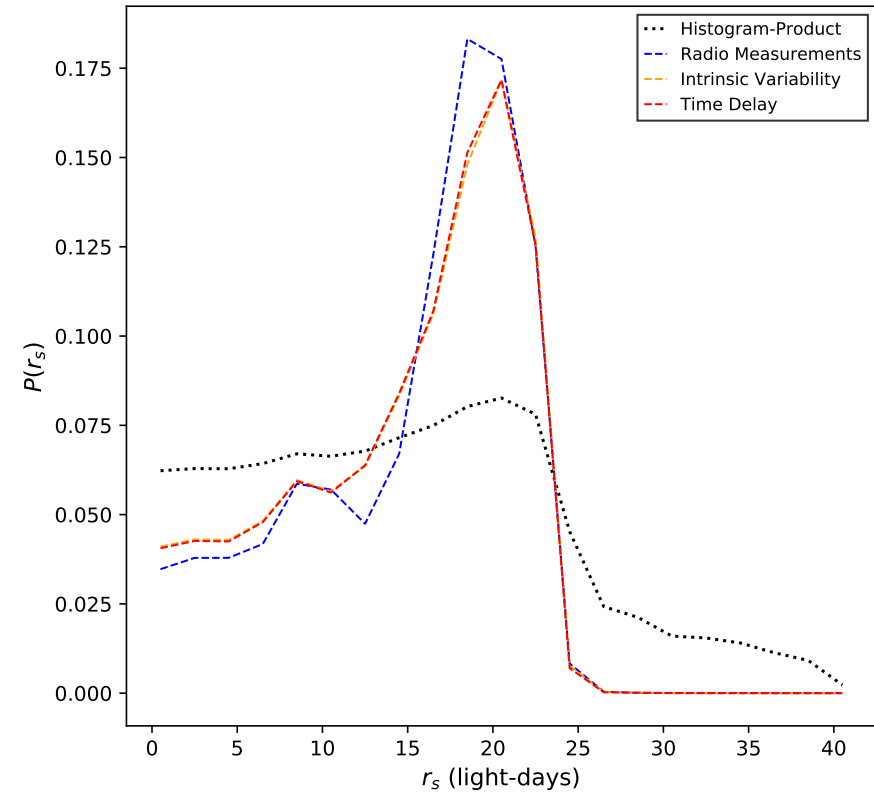

Fig. 6. Probability density distribution of the source size $r_{s}$ using a time delay of $\Delta t=417$ days, a radio-flux ratio of $B / A=0.74$, a linear interpolation of image A's light curve as an intrinsic variability model, and the lens model parameters of Mediavilla et al. (2009) (dotted black line). The dashed lines show the PDFs for various model/data-related analyses as indicated by the legend. These are obtained by multiplying single probability distributions corresponding to different time delays (red), models for the intrinsic variability (orange; see Table 4), and radio measurements (blue; see Table 6). We note that the red and orange distributions overlap.

tion probability can be directly inferred from the histogram of microlensing magnifications corresponding to image B. As a cross-check, we repeated the calculations considering only the magnification map for image $\mathrm{B}$, obtaining the same result.

\subsection{Probability density function of the source size}

To evaluate the impact that changes in the previously discussed values/models can have on the results, we multiplied all the possible probability distributions for each source of uncertainty (see Fig. 6). That means, in the case of the intrinsic variability, we multiplied five probability distributions; for the radio/BEL measurements, we multiplied twelve probability distributions; and so on. In Table 8, we summarize the contributions of the different sources of uncertainty on the size estimates and list their deviation from the inferred disk size when using the most recent values for both, the time delay ( $\Delta t=417$ days), and the radio flux ratio $(B / A=0.74)$, a linear interpolation for the image's A light curve as an intrinsic model, and the lens model parameters of Mediavilla et al. (2009). The uncertainties in modeling the intrinsic variability as well as the uncertainties in the time delay have no significant influence on the size. With regard to the baseline of no microlensing, assuming very conservative errors, we obtain an increase of $\sim 3 \%$ in the estimate of the size. Thus, we get $\sim 30 \%$ smaller sizes for the accretion disk using the lens model of Pelt et al. (1998), and $20 \%$ bigger sizes when using a different fraction of mass in stars for both images. Finally, we obtain a half-light radius of $R_{1 / 2}=17.6 \pm 6.1 \sqrt{M / 0.3 M_{\odot}}$ lt-days for the region emitting the R-band emission using $\sqrt{\sum_{i} \Delta R_{1 / 2}{ }_{i}^{2}}$ from Table 8 to estimate the uncertainty in size (i.e., the $1 \sigma$ 
variation of the peak of the probability distributions in each direction).

\subsection{Impact of the effective velocity on the size estimate}

The information on the observed light curve can be better extracted by full fitting procedures (Kochanek 2004; Hainline et al. 2012; Cornachione et al. 2020). This procedure is beyond the scope of this paper, yet it is not the only possible approach to use the observed light curve. Even if we do not use the time-ordered series of data, we have presented a simple, fast statistical approach to extract information on the source size from the light curve (e.g., Fian et al. 2016). Nevertheless, the effective velocity of the source can still affect the timescale on which points in the observed light curves have to be compared with our models. To take this effect into account, we tested the influence of the velocity on our size estimate by sampling (i.e., averaging using a certain window) the experimental light curves with the time window corresponding to the magnification map pixel size and the effective velocity for the source trajectory. Hainline et al. (2012) constructed the effective source plane (Einstein) velocity, $v_{\mathrm{e}}$, using the method described in Kochanek (2004), applying the measured stellar velocity dispersion for the lens galaxy $\left(\sigma_{\star}=288 \pm 9 \mathrm{~km} \mathrm{~s}^{-1}\right.$; Tonry \& Franx 1999), and obtaining the dispersion of the peculiar velocity distribution at the redshifts of the quasar and the lens galaxy from the power-law fits by Mosquera et al. (2011) to the peculiar velocity models of Tinker et al. (2012). As a consequence of the low intrinsic variability of Q $0957+561$, coupled with the relatively low amplitude of the microlensing signal, they obtained a wide velocity distribution with a median of $1600 \mathrm{~km} \mathrm{~s}^{-1}$ and a $68 \%$ confidence range of $600 \mathrm{~km} \mathrm{~s}^{-1}<v_{\mathrm{e}}<3500 \mathrm{~km} \mathrm{~s}^{-1}$. The median velocity corresponds to a time window of 1.2 months for a pixel size of 0.2 lt-days ( $\sim 200$ points in the 21 years long light curves). The $+1 \sigma$ velocity coincides with a time window of 0.54 months (i.e., $\sim 480$ data points), and the $-1 \sigma$ velocity with a time window of 3.3 months ( $\sim 70$ data points). We built three experimental histograms (see Fig. 7) of the (according to the velocity averaged) microlensing difference light curves and compared them with the simulated histograms for different sizes of $r_{s}$, as described in Sect. 4. We found that the overall shape of the experimental histograms stays the same and that the velocity does not induce significant changes in the size estimates (with a maximum change of $\sim 0.1$ lt-days).

\section{Conclusions}

We studied 21 years of monitoring data for the lensed images of the twin quasar Q $0957+561$, which so far are the longest available light curves of a gravitational lens system. Unlike most other known lens systems, photometric monitoring of this object is relatively easy since the system is relatively bright $(I=16)$ and because of its wide image separation $\left(\sim 6^{\prime \prime}\right)$. We used the GLENDAMA light curves (Gil-Merino et al. 2018) to obtain the accretion disk size, which with more than thousand epochs of observation significantly extend the time coverage of previous works. Taking image A, which is less affected by microlensing, as a reference and using the experimental time delays inferred by Shalyapin et al. (2008), we removed the intrinsic variability from light curve $\mathrm{B}$ in the overlapping region. Using the radio-flux ratio between components $\mathrm{A}$ and $\mathrm{B}$ measured by Haarsma et al. (1999) as a baseline for no microlensing, we finally obtained the microlensing light curve $\mathrm{B}-\mathrm{A}$. We found microlensing of up to $0.5 \mathrm{mag}$ in the residuals of recent years and we used the

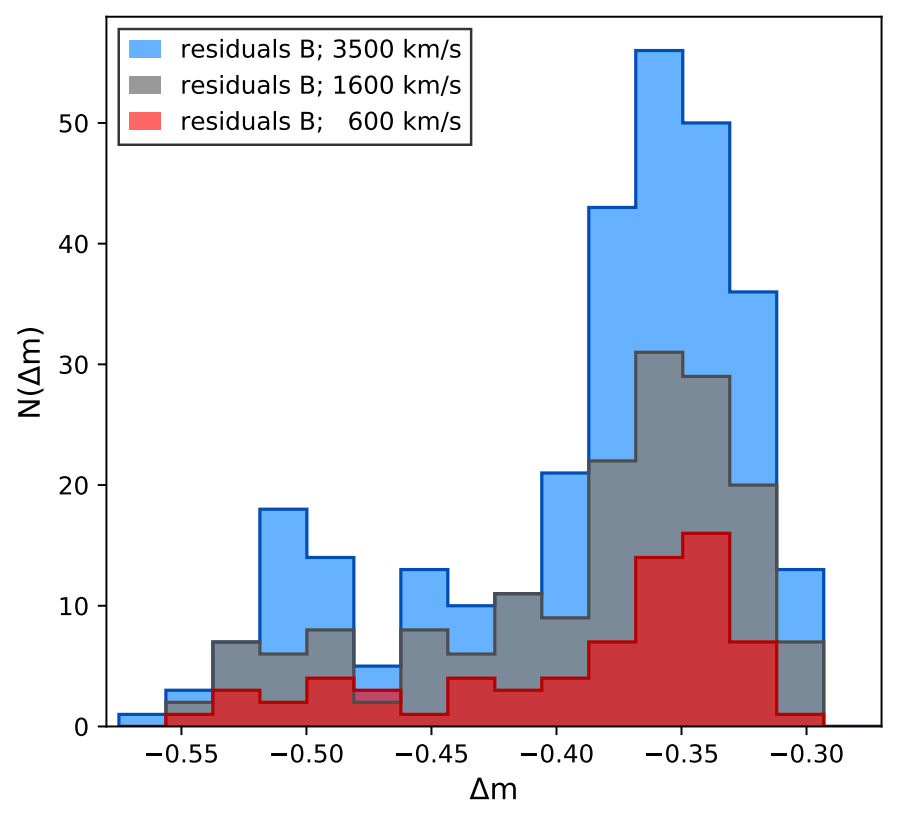

Fig. 7. Microlensing frequency distributions obtained from the (according to the effective velocity averaged) difference light curves for $v_{\mathrm{e}}=$ $1600 \mathrm{~km} \mathrm{~s}^{-1}$ (gray), $v_{\mathrm{e}}=600 \mathrm{~km} \mathrm{~s}^{-1}$ (red), and $v_{\mathrm{e}}=3500 \mathrm{~km} \mathrm{~s}^{-1}$ (blue).

statistics of microlensing during all available seasons to infer probabilistic distributions for the source size. Using the product of the observed and modeled microlensing histograms, we obtained a half-light radius of $R_{1 / 2}=17.6 \pm 6.1 \sqrt{M / 0.3 M_{\odot}}$ ltdays. According to Table 8 , uncertainties in the faction of mass in stars and in the macromodel are the dominant sources of error. However, uncertainties corresponding to these values in the error budget are probably conservative upper limits. In Sect. 5.1, we see that there is no significant dependence on the model used to simulate the quasar's intrinsic variability. Thus, small changes in the estimation of the time delay between images have no effect on the source size (see Sect. 5.2). This supports both the robustness of the method and the limited impact of uncertainties on the accretion disk size.

Our estimate for the size is significantly greater than the average determinations obtained for a sample of lensed quasars by Jiménez-Vicente et al. $(2012,2014)\left(R_{1 / 2}(2558 \AA) \sim 8\right.$ ltdays and $R_{1 / 2}(2558 \AA) \sim 10$ lt-days, respectively) when scaling the rest wavelength (from $1736 \AA$ and $1026 \AA$, respectively) to $2558 \AA$ (using $R_{1 / 2}=\left(\lambda_{0} / \lambda\right)^{p} R_{1 / 2}(\lambda)$ ) for microlenses with a mean mass of $M=0.3 M_{\odot}$ and assuming $\alpha=10 \%$. Comparing with the average accretion disk size obtained by Jiménez-Vicente et al. (2015b) when using a more realistic value for the fraction of mass in stars $(\alpha=20 \%)$, their reported values of $\sim 10$ lt-days (maximum likelihood) and $\sim 8$ lt-days (Bayesian) at $\lambda_{\text {rmrest }}=1736 \AA$ correspond to $\sim 16$ lt-days and $\sim 13$ lt-days at $2558 \AA$ (for microlenses with a mean mass of $M=0.3 M_{\odot}$ ), matching our estimate for the disk size in Q $0957+561$. Our result appears to be consistent within errors with the R-band half-light radius of Hainline et al. (2012) $\left(R_{1 / 2}=12.2_{-8.3}^{+26.4}\right.$ lt-days). Furthermore, our results are in excellent agreement with the continuum size recently predicted by Cornachione et al. (2020) $\left(R_{1 / 2}=17.6_{-13.4}^{+23.8}\right.$ at $\left.\lambda_{\text {rest }}=2558 \AA\right)$ using the light curve fitting method (see Kochanek 2004). Thus, we observe that the microlensing size is significantly larger than the prediction of the thin disk theory, also found for other lensed quasars by 
Pooley et al. (2007), Morgan et al. (2010), and Blackburne et al. (2011). We note that the broad-line region could contribute substantially to the continuum level around the Mg II line due to the underlying iron blends, the Balmer recombination edge, and the $\mathrm{Mg}$ II line itself. The degree to which recent findings for lowluminosity sources are also relevant for high-luminosity quasars is still unclear (see, e.g., Chelouche et al. 2019; Korista \& Goad 2001, 2019; Lawther et al. 2018).

Acknowledgements. We thank the anonymous referee for the helpful comments, and constructive remarks on this manuscript. We thank the GLENDAMA project for making publicly available the monitoring data of Q $0957+561$. C.F. gratefully acknowledges the financial support from Tel Aviv University and University of Haifa through a DFG grant HA3555-14/1. E.M. and J.A.M are supported by the Spanish MINECO with the grants AYA2016- 79104-C3-1-P and AYA2016-79104-C3-3-P. J.A.M. is also supported from the Generalitat Valenciana project of excellence Prometeo/2020/085. J.J.V. is supported by the project AYA2017-84897-P financed by the Spanish Ministerio de Economía y Competividad and by the Fondo Europeo de Desarrollo Regional (FEDER), and by project FQM-108 financed by Junta de Andalucía. K. R. acknowledges support from the Swiss National Science Foundation (SNSF). V.M. acknowledges the support of Centro de Astrofísica de Valparaíso.

\section{References}

Birrer, S., Shajib, A. J., Galan, A., et al. 2020, A\&A, 643, A165

Blackburne, J. A., Pooley, D., Rappaport, S., \& Schechter, P. L. 2011, ApJ, 729

Blackburne, J. A., Kochanek, C. S., Chen, B., Dai, X., \& Chartas, G. 2014, ApJ, 789,125

Blackburne, J. A., Kochanek, C. S., Chen, B., Dai, X., \& Chartas, G. 2015, ApJ, 798,95

Chang, K., \& Refsdal, S. 1979, Nature, 282, 56

Chang, K., \& Refsdal, S. 1984, A\&A, 132, 168

Chartas, G., Falco, E., Forman, W., et al. 1995, ApJ, 445, 140

Chelouche, D., Pozo Nuñez, F., \& Kaspi, S. 2019, Nat. Astron., 3, 251

Colley, W. N., Schild, R. E., Abajas, C., et al. 2003, ApJ, 587, 71

Conner, S. R., Lehar, J., \& Burke, B. F. 1992, ApJ, 387, L61

Cornachione, M. A., Morgan, C. W., Burger, H. R., et al. 2020, ApJ, 905, 7

Fadely, R., Keeton, C. R., Nakajima, R., \& Bernstein, G. M. 2010, ApJ, 711, 246

Fian, C., Mediavilla, E., Hanslmeier, A., et al. 2016, ApJ, 830, 149

Fian, C., Mediavilla, E., Jiménez-Vicente, J., Muñoz, J. A., \& Hanslmeier, A. 2018, ApJ, 869, 132

Garrett, M. A. 1990, Ph.D. Thesis, University of Manchester, UK

Garrett, M. A., Calder, R. J., Porcas, R. W., et al. 1994, MNRAS, 270, 457

Gil-Merino, R., Goicoechea, L. J., Shalyapin, V. N., \& Oscoz, A. 2018, A\&A, 616, A118

Goicoechea, L. J., Gil-Merino, R., \& Ullán, A. 2005, MNRAS, 360, L60

Goicoechea, L. J., Shalyapin, V. N., Koptelova, E., et al. 2008, New Astron., 13, 182

Gorenstein, M. V., Cohen, N. L., Shapiro, I. I., et al. 1988, ApJ, 334, 42 Guerras, E., Mediavilla, E., Jimenez-Vicente, J., et al. 2013, ApJ, 764, 160 Haarsma, D. B., Hewitt, J. N., Lehár, J., \& Burke, B. F. 1999, ApJ, 510, 64 Hainline, L. J., Morgan, C. W., Beach, J. N., et al. 2012, ApJ, 744, 104 Jiménez-Vicente, J., Mediavilla, E., Muñoz, J. A., \& Kochanek, C. S. 2012, ApJ, 751,106

Jiménez-Vicente, J., Mediavilla, E., Kochanek, C. S., et al. 2014, ApJ, 783, 47 Jiménez-Vicente, J., Mediavilla, E., Kochanek, C. S., \& Muñoz, J. A. 2015a ApJ, 806, 251
Jiménez-Vicente, J., Mediavilla, E., Kochanek, C. S., \& Muñoz, J. A. 2015b, ApJ, 799, 149

Kochanek, C. S. 2004, ApJ, 605, 58

Korista, K. T., \& Goad, M. R. 2001, ApJ, 553, 695

Korista, K. T., \& Goad, M. R. 2019, MNRAS, 489, 5284

Kundić, T., Turner, E. L., Colley, W. N., et al. 1997, ApJ, 482, 75

Lawther, D., Goad, M. R., Korista, K. T., Ulrich, O., \& Vestergaard, M. 2018, MNRAS, 481, 533

Mediavilla, E., Muñoz, J. A., Lopez, P., et al. 2006, ApJ, 653, 942

Mediavilla, E., Muñoz, J. A., Falco, E., et al. 2009, ApJ, 706, 1451

Mediavilla, E., Mediavilla, T., Muñoz, J. A., et al. 2011, ApJ, 741, 42

Mediavilla, E., Jimenez-Vicente, J., Muñoz, J. A., Mediavilla, T., \& Ariza, O. 2015, ApJ, 798, 138

Mediavilla, E., Jimenez-Vicente, J., Muñoz, J. A., \& Battaner, E. 2016, ApJ, 832, 46

Metcalf, R. B., \& Zhao, H. 2002, ApJ, 567, L5

Morgan, C. W., Kochanek, C. S., Morgan, N. D., \& Falco, E. E. 2010, ApJ, 712, 1129

Morgan, C. W., Hyer, G. E., Bonvin, V., et al. 2018, ApJ, 869, 106

Mortonson, M. J., Schechter, P. L., \& Wambsganss, J. 2005, ApJ, 628, 594

Mosquera, A. M., \& Kochanek, C. S. 2011, ApJ, 738, 96

Mosquera, A. M., Muñoz, J. A., \& Mediavilla, E. 2009, ApJ, 691, 1292

Mosquera, A. M., Muñoz, J. A., Mediavilla, E., \& Kochanek, C. S. 2011, ApJ, 728,145

Mosquera, A. M., Kochanek, C. S., Chen, B., et al. 2013, ApJ, 769, 53

Motta, V., Mediavilla, E., Falco, E., \& Muñoz, J. A. 2012, ApJ, 755, 82

Muñoz, J. A., Vives-Arias, H., Mosquera, A. M., et al. 2016, ApJ, 817, 155

Oscoz, A., Serra-Ricart, M., Goicoechea, L. J., Buitrago, J., \& Mediavilla, E. 1996, ApJ, 470, L19

Oscoz, A., Mediavilla, E., Goicoechea, L. J., Serra-Ricart, M., \& Buitrago, J. 1997, ApJ, 479, L89

Oscoz, A., Alcalde, D., Serra-Ricart, M., et al. 2001, ApJ, 552, 81

Oscoz, A., Alcalde, D., Serra-Ricart, M., Mediavilla, E., \& Muñoz, J. A. 2002, ApJ, 573, L1

Ovaldsen, J. E., Teuber, J., Schild, R. E., \& Stabell, R. 2003, A\&A, 402, 891

Paraficz, D., Hjorth, J., Burud, I., Jakobsson, P., \& Elíasdóttir, Á. 2006, A\&A, 455, L1

Pelt, J., Kayser, R., Refsdal, S., \& Schramm, T. 1996, A\&A, 305, 97

Pelt, J., Schild, R., Refsdal, S., \& Stabell, R. 1998, A\&A, 336, 829

Pooley, D., Blackburne, J. A., Rappaport, S., \& Schechter, P. L. 2007, ApJ, 661, 19

Popović, L. Č., Afanasiev, V. L., Shablovinskaya, E. S., Ardilanov, V. I., \& Savić, D. 2021, A\&A, 647, A98

Refsdal, S. 1964, MNRAS, 128, 307

Rusu, C. E., Wong, K. C., Bonvin, V., et al. 2020, MNRAS, 498, 1440

Schechter, P. L., \& Wambsganss, J. 2002, ApJ, 580, 685

Schild, R. E., \& Smith, R. C. 1991, AJ, 101, 813

Schild, R., \& Thomson, D. J. 1997, AJ, 113, 130

Schild, H., Smith, L. J., \& Willis, A. J. 1990, A\&A, 237, 169

Schmidt, R., \& Wambsganss, J. 1998, A\&A, 335, 379

Serra-Ricart, M., Oscoz, A., Sanchís, T., et al. 1999, ApJ, 526, 40

Shalyapin, V. N., Goicoechea, L. J., Koptelova, E., Ullán, A., \& Gil-Merino, R. 2008, A\&A, 492, 401

Shalyapin, V. N., Goicoechea, L. J., \& Gil-Merino, R. 2012, A\&A, 540, A132

Sluse, D., Kishimoto, M., Anguita, T., Wucknitz, O., \& Wambsganss, J. 2013, A\&A, 553, A53

Tinker, J. L., Sheldon, E. S., Wechsler, R. H., et al. 2012, ApJ, 745, 16

Tonry, J. L., \& Franx, M. 1999, ApJ, 515, 512

Wambsganss, J. 2006, ArXiv e-prints [arXiv:astro-ph/0604278]

Wambsganss, J., Schmidt, R. W., Colley, W., Kundić, T., \& Turner, E. L. 2000, A\&A, 362, L37

Wong, K. C., Suyu, S. H., Chen, G. C. F., et al. 2020, MNRAS, 498, 1420

Young, P. 1981, ApJ, 244, 756 